\title{
Sectional Invariants of Scrolls Over a Smooth Projective Variety
}

\author{
YoSHIAKI FuKUma $(*)$
}

ABSTRACT - Let $X$ be a smooth complex projective variety of dimension $n$ and let $\mathcal{E}$ be an ample vector bundle of rank $r$ on $X$. Then we calculate the $i$ th sectional Euler number $e_{i}\left(\mathbb{P}_{X}(\mathcal{E}), H(\mathcal{E})\right)$ and the $i$ th sectional Betti number $b_{i}\left(\mathbb{P}_{X}(\mathcal{E}), H(\mathcal{E})\right)$ for $i \geq 2 n-3$ or $i=1$, and the $i$ th sectional Hodge number of type $(j, i-j)$ $h_{i}^{j, i-j}\left(\mathbb{P}_{X}(\mathcal{E}), H(\mathcal{E})\right)$ for $i \geq 2 n-1$ and $0 \leq j \leq i$, where $\mathbb{P}_{X}(\mathcal{E})$ is the projective space bundle associated with $\mathcal{E}$ and $H(\mathcal{E})$ is its tautological line bundle. Moreover we define a new invariant $v(X, \mathcal{E})$ of $(X, \mathcal{E})$ for $r \geq n-1$. This invariant is thought to be a generalization of curve genus. We will investigate some properties of this invariant.

\section{Introduction.}

Let $X$ be a projective variety of dimension $n$ defined over the field of complex numbers, and let $L$ be an ample (resp. a nef and big) line bundle on $X$. Then $(X, L)$ is called a polarized (resp. quasi-polarized) variety. If $X$ is smooth, then we say that $(X, L)$ is a polarized (resp. quasi-polarized) manifold. In order to study polarized varieties, it is important to use an invariant of $(X, L)$. There are the following three invariants of $(X, L)$ which are well-known.

- The degree $L^{n}$.

- The sectional genus $g(L)$.

- The $\Delta$-genus $\Delta(L)$.

By using these invariants, many authors studied polarized varieties. In particular, P. Ionescu classified polarized manifolds by the degree under the assumption that $L$ is very ample with $L^{n} \leq 8$ ([13], [14], and [15]), and

(*) Indirizzo dell'A.: Department of Natural Science, Faculty of Science, Kochi University, Akebono-cho, Kochi 780-8520, Japan.

E-mail: fukuma@kochi-u.ac.jp 
T. Fujita classified polarized varieties by the 4 -genus and the sectional genus ([3]).

In [5], in order to study polarized varieties more deeply, the author introduced the notion of the $i$ th sectional geometric genus $g_{i}(X, L)$ of $(X, L)$ for every integer $i$ with $0 \leq i \leq n$. This is a generalization of the degree and the sectional genus of $(X, L)$. Namely $g_{0}(X, L)=L^{n}$ and $g_{1}(X, L)=g(L)$.

Here we recall the reason why this invariant is called the $i$ th sectional geometric genus. Let $(X, L)$ be a polarized manifold of dimension $n \geq 2$ with $\mathrm{Bs}|L|=\emptyset$, where $\mathrm{Bs}|L|$ is the base locus of the complete linear system $|L|$. Let $i$ be an integer with $1 \leq i \leq n$. Let $X_{n-i}$ be the transversal intersection of general $n-i$ members of $|L|$. In this case $X_{n-i}$ is a smooth projective variety of dimension $i$. Then we can prove that $g_{i}(X, L)=h^{i}\left(\mathcal{O}_{X_{n-i}}\right)$, that is, $g_{i}(X, L)$ is the geometric genus of $X_{n-i}$.

Hence we can expect that $g_{i}(X, L)$ has analogous properties of the geometric genus of $i$-dimensional varieties.

In [6] and [7], we introduced the notion of the ith sectional $H$-arithmetic genus $\chi_{i}^{H}(X, L)$ of $(X, L)$. By definition we can prove that if $\mathrm{Bs}|L|=\emptyset$, then $\chi_{i}^{H}(X, L)=\chi\left(\mathcal{O}_{X_{n-i}}\right)$, where $X_{n-i}$ is the transversal intersection of general $n-i$ members of $|L|$. Namely $\chi_{i}^{H}(X, L)$ is the Euler-Poincaré characteristic of the structure sheaf of $X_{n-i} \cdot\left(\chi\left(\mathcal{O}_{X_{n-i}}\right)\right.$ is called the arithmetic genus of $X_{n-i}$ in the sense of Hirzebruch. (See [12, 15.5, Section 15, Chapter IV]. We also call $\chi\left(\mathcal{O}_{X_{n-i}}\right)$ the H-arithmetic genus of $X_{n-i}$. $)$

In [8], we also introduced some $i$ th sectional invariants of $(X, L)$, that is, the $i$ th sectional Euler number $e_{i}(X, L)$, the $i$ th sectional Betti number $b_{i}(X, L)$ and the $i$ th sectional Hodge number $h_{i}^{j, i-j}(X, L)$ of type $(j, i-j)$ for every integer $j$ with $0 \leq j \leq i$, and we investigated some properties of these. In particular we proved that polarized manifolds' version of the Hodge duality and the Hodge decomposition hold (see [8, Theorem 3.1]).

In this paper we consider the $i$ th sectional Euler number and the $i$ th sectional Betti number of scrolls over a smooth projective variety. In this paper we say that a polarized manifold $(P, H)$ is a scroll over a smooth projective variety $X$ if there exists an ample vector bundle $\mathcal{E}$ on $X$ such that $(P, H) \cong\left(\mathbb{P}_{X}(\mathcal{E}), H(\mathcal{E})\right)$, where $H(\mathcal{E})$ is the tautological line bundle on $\mathbb{P}_{X}(\mathcal{E})$.

In section 3 , we calculate $e_{i}\left(\mathbb{P}_{X}(\mathcal{E}), H(\mathcal{E})\right)$ and $b_{i}\left(\mathbb{P}_{X}(\mathcal{E}), H(\mathcal{E})\right)$ for $i \geq 2 n-3$ and $i=1$ (see Theorems 3.1 and 3.2). We also calculate $h_{i}^{j, i-j}\left(\mathbb{P}_{X}(\mathcal{E}), H(\mathcal{E})\right)$ for $i \geq 2 n-1$ and $0 \leq j \leq i$ (see Theorem 3.3). In particular, by using these results, we can calculate these invariants of $\left(\mathbb{P}_{X}(\mathcal{E}), H(\mathcal{E})\right.$ ) completely for $n=1$ or 2 (see Corollaries 3.1, 3.3 and 3.4). 
In section 4 , we will define a new invariant of generalized polarized manifolds. Here a generalized polarized manifold means the pair $(X, \mathcal{E})$ where $X$ is a smooth projective variety and $\mathcal{E}$ is an ample vector bundle on $X$. Let $r:=\operatorname{rank}(\mathcal{E})$. Here we state the history of invariants of $(X, \mathcal{E})$. First in [2], Fujita introduced the $c_{1}$-sectional genus and the $\mathcal{O}(1)$-sectional genus of $(X, \mathcal{E})$. Next, in [1], for the case where $r=n-1$, Ballico defined an invariant of $(X, \mathcal{E})$ which is called the curve genus $\operatorname{cg}(X, \mathcal{E})$ of $(X, \mathcal{E})$ (see Definition 2.3), and several authors (in particular Lanteri, Maeda, Sommese, and so on) studied this invariant.

As a generalization of the curve genus, for any ample vector bundle $\mathcal{E}$ with $r \leq n-1$, Ishihara ([16, Definition 1.1]) defined an invariant $g(X, \mathcal{E})$, which is called the $c_{r}$-sectional genus of $(X, \mathcal{E})$, and in [9] we investigated some properties about $g(X, \mathcal{E})$. We note that if $n-r=1$, then $g(X, \mathcal{E})$ is the curve genus. This invariant means the following: If a general element of $H^{0}(\mathcal{E})$ has a zero locus $Z$ which is smooth of expected dimension $n-r$, then $g(X, \mathcal{E})=g\left(Z,\left.\operatorname{det} \mathcal{E}\right|_{Z}\right)$, that is, $g(X, \mathcal{E})$ is the sectional genus of $\left(Z\right.$, $\left.\left.\operatorname{det} \mathcal{E}\right|_{Z}\right)$. Recently Fusi and Lanteri generalized this invariant. See [11] for detail.

In this paper, we will introduce a new invariant $v(X, \mathcal{E})$ of generalized polarized manifolds $(X, \mathcal{E})$ with $r \geq n-1$, which is defined by using a result in section 3 (see Definition 4.1). Here we note that $v(X, \mathcal{E})$ is equal to the curve genus if $r=n-1$. We will investigate $v(X, \mathcal{E})$ and give some results about this invariant (see Theorems 4.1 and 4.2, and Proposition 4.1).

The author would like to thank the referee for giving useful comments and suggestions.

\section{Notation and Conventions.}

We say that $X$ is a variety if $X$ is an integral separated scheme of finite type. In particular $X$ is irreducible and reduced if $X$ is a variety. Varieties are always assumed to be defined over the field of complex numbers. In this article, we shall study mainly a smooth projective variety. The words "line bundles" and "Cartier divisors" are used interchangeably. The tensor products of line bundles are denoted additively.

$\mathcal{O}(D)$ : invertible sheaf associated with a Cartier divisor $D$ on $X$.

$\mathcal{O}_{X}$ : the structure sheaf of $X$.

$\chi(\mathcal{F})$ : the Euler-Poincare characteristic of a coherent sheaf $\mathcal{F}$.

$h^{i}(\mathcal{F}):=\operatorname{dim} H^{i}(X, \mathcal{F})$ for a coherent sheaf $\mathcal{F}$ on $X$.

$h^{i}(D):=h^{i}(\mathcal{O}(D))$ for a Cartier divisor $D$.

$q(X)\left(=h^{1}\left(\mathcal{O}_{X}\right)\right):$ the irregularity of $X$. 
$b_{i}(X):=\operatorname{dim} H^{i}(X, \mathrm{C})$.

$K_{X}$ : the canonical divisor of $X$.

$\mathbb{P}^{n}$ : the projective space of dimension $n$.

$Q^{n}$ : a smooth quadric hypersurface in $\mathbb{P}^{n+1}$.

$\sim($ or $=)$ : linear equivalence.

$\operatorname{det}(\mathcal{E}):=\wedge^{r} \mathcal{E}$, where $\mathcal{E}$ is a vector bundle of rank $r$ on $X$.

$\mathbb{P}_{X}(\mathcal{E})$ : the projective space bundle associated with a vector bundle $\mathcal{E}$ on $X$.

$H(\mathcal{E})$ : the tautological line bundle on $\operatorname{P}_{X}(\mathcal{E})$.

$\mathcal{E}^{\vee}$ : the dual of a vector bundle $\mathcal{E}$.

$c_{i}(\mathcal{E})$ : the $i$-th Chern class of a vector bundle $\mathcal{E}$.

$c_{i}(X):=c_{i}\left(\mathcal{T}_{X}\right)$, where $\mathcal{T}_{X}$ is the tangent bundle of a smooth projective variety $X$.

For a real number $m$ and a non-negative integer $n$, let

$$
\begin{aligned}
{[m]^{n}: } & =\left\{\begin{array}{cc}
m(m+1) \cdots(m+n-1) & \text { if } n \geq 1, \\
1 & \text { if } n=0 .
\end{array}\right. \\
{[m]_{n}: } & =\left\{\begin{array}{cc}
m(m-1) \cdots(m-n+1) & \text { if } n \geq 1, \\
1 & \text { if } n=0 .
\end{array}\right.
\end{aligned}
$$

Then for $n$ fixed, $[m]^{n}$ and $[m]_{n}$ are polynomials in $m$ whose degree are $n$. For any non-negative integer $n$,

$$
n !:=\left\{\begin{array}{cc}
{[n]_{n}} & \text { if } n \geq 1 \\
1 & \text { if } n=0 .
\end{array}\right.
$$

Assume that $m$ and $n$ are integers with $n \geq 0$. Then we put

$$
\left(\begin{array}{c}
m \\
n
\end{array}\right):=\frac{[m]_{n}}{n !}
$$

We note that $\left(\begin{array}{c}m \\ n\end{array}\right)=0$ if $0 \leq m<n$, and $\left(\begin{array}{c}m \\ 0\end{array}\right)=1$.

\section{Preliminaries.}

Notation 2.1. (1) Let $Y$ be a smooth projective variety of dimension $i$, let $\mathcal{T}_{Y}$ be the tangent bundle of $Y$, and let $\Omega_{Y}\left(=\Omega_{Y}^{1}\right)$ be the dual bundle of $\mathcal{T}_{Y}$ and $\Omega_{Y}^{j}:=\wedge^{j} \Omega_{Y}$. For every integer $j$ with $0 \leq j \leq i$, we put

$$
\begin{aligned}
h_{i, j}\left(c_{1}(Y), \cdots, c_{i}(Y)\right) & :=\chi\left(\Omega_{Y}^{j}\right) \\
& =\int_{Y} \operatorname{ch}\left(\Omega_{Y}^{j}\right) \operatorname{Td}\left(\mathcal{T}_{Y}\right) .
\end{aligned}
$$


(Here $\operatorname{ch}\left(\Omega_{Y}^{j}\right)$ (resp. $\operatorname{Td}\left(\mathcal{T}_{Y}\right)$ ) denotes the Chern character of $\Omega_{Y}^{j}$ (resp. the Todd class of $\mathcal{T}_{Y}$ ). See [10, Example 3.2.3 and Example 3.2.4].)

(2) Let $(M, L)$ be a polarized manifold of dimension $m$. For every integers $i$ and $j$ with $0 \leq j \leq i \leq m$, we put

$$
\begin{gathered}
C_{j}^{i}(M, L):=\sum_{l=0}^{j}(-1)^{l}\left(\begin{array}{c}
m-i+l-1 \\
l
\end{array}\right) c_{j-l}(M) L^{l}, \\
w_{i}^{j}(M, L):=h_{i, j}\left(C_{1}^{i}(M, L), \cdots, C_{i}^{i}(M, L)\right) L^{n-i} .
\end{gathered}
$$

(3) Let $M$ be a smooth projective variety of dimension $m$. For every integers $i$ and $j$ with $0 \leq j \leq i \leq m$, we put

$$
\begin{aligned}
& H_{1}(i, j):= \begin{cases}\sum_{s=0}^{i-j-1}(-1)^{s} h^{s}\left(\Omega_{M}^{j}\right) & \text { if } j \neq i, \\
0 & \text { if } j=i,\end{cases} \\
& H_{2}(i, j):= \begin{cases}\sum_{t=0}^{j-1}(-1)^{i-t} h^{t}\left(\Omega_{M}^{i-j}\right) & \text { if } j \neq 0, \\
0 & \text { if } j=0 .\end{cases}
\end{aligned}
$$

Definition 2.1. (See [8, Definition 3.1].) Let $(M, L)$ be a polarized manifold of dimension $m$, and let $i$ and $j$ be integers with $0 \leq i \leq m$ and $0 \leq j \leq i$.

(1) The ith sectional Euler number $e_{i}(M, L)$ of $(M, L)$ is defined by the following:

$$
e_{i}(M, L):=\sum_{l=0}^{i}(-1)^{l}\left(\begin{array}{c}
m-i+l-1 \\
l
\end{array}\right) c_{i-l}(M) L^{m-i+l}
$$

(2) The ith sectional Betti number $b_{i}(M, L)$ of $(M, L)$ is defined by the following:

$$
b_{i}(M, L):= \begin{cases}e_{0}(M, L) & \text { if } i=0, \\ (-1)^{i}\left(e_{i}(M, L)-\sum_{j=0}^{i-1} 2(-1)^{j} b_{j}(M)\right) & \text { if } 1 \leq i \leq m .\end{cases}
$$

(3) The ith sectional Hodge number $h_{i}^{j, i-j}(M, L)$ of type $(j, i-j)$ of 
$(M, L)$ is defined by the following:

$$
h_{i}^{j, i-j}(M, L):=(-1)^{i-j}\left\{w_{i}^{j}(M, L)-H_{1}(i, j)-H_{2}(i, j)\right\} .
$$

REMARK 2.1. (1) If $i=0$, then

$$
e_{0}(M, L)=b_{0}(M, L)=h_{0}^{0,0}(M, L)=L^{m} .
$$

(2) If $i=1$, then

$$
\begin{aligned}
e_{1}(M, L) & =2-2 g(L), \\
b_{1}(M, L) & =2 g(L), \\
h_{1}^{1,0}(M, L) & =h_{1}^{0,1}(M, L)=g(L) .
\end{aligned}
$$

(3) If $i=m$, then

$$
\begin{gathered}
e_{m}(M, L)=e(M), \\
b_{m}(M, L)=b_{m}(M), \\
h_{m}^{j, m-j}(M, L)=h^{j, m-j}(M), \\
h_{m}^{m-j, j}(M, L)=h^{m-j, j}(M) .
\end{gathered}
$$

Proposition 2.1. Let $(M, L)$ be a polarized manifold of dimension $m$.

(1) For every integer $i$ with $0 \leq i \leq m$, the following hold:

(1.1) $b_{i}(M, L)=\sum_{k=0}^{i} h_{i}^{k, i-k}(M, L)$.

(1.2) $h_{i}^{j, i-j}(M, L)=h_{i}^{i-j, j}(M, L)$.

(1.3) $h_{i}^{i, 0}(M, L)=h_{i}^{0, i}(M, L)=g_{i}(M, L)$.

(2) Assume that $L$ is base point free. Then for every integers $i$ and $j$ with $1 \leq i \leq m$ and $0 \leq j \leq i$ the following hold.

(2.1) $\quad b_{i}(M, L) \geq b_{i}(M)$.

(2.2) $\quad h_{i}^{j, i-j}(M, L) \geq h^{j, i-j}(M)$.

Proof. See [8, Theorem 3.1 (3.1.1), (3.1.3), (3.1.4) and Proposition 3.3 (2) and (3)]. 
Proposition 2.2. For every integers $a, k, l$ and $r$ with $0 \leq l$,

$$
\sum_{j=0}^{l}(-1)^{j}\left(\begin{array}{c}
r+j-a \\
j
\end{array}\right)\left(\begin{array}{c}
r-k \\
l-j
\end{array}\right)=(-1)^{l}\left(\begin{array}{c}
k-a+l \\
l
\end{array}\right) .
$$

Proof. See [8, Proposition 2.5].

Notation 2.2. Let $X$ be a smooth projective variety of dimension $n \geq 1$ and let $\mathcal{E}$ be an ample vector bundle of rank $r$ on $X$. We put $P:=\mathbb{P}_{X}(\mathcal{E})$, $H:=H(\mathcal{E})$ and $m:=\operatorname{dim} P$. Then $m=n+r-1$. In this paper we assume that $r \geq 2$.

Remark 2.2. Let $X, \mathcal{E}, P, H, m, n$ and $r$ be as in Notation 2.2.

(1) By [18, (2.1) Proposition], we have

$$
b_{j}(P)=b_{j}(X)+b_{j-2}(X)+\cdots+b_{j-2 r+2}(X) .
$$

(2) Let $i$ be an integer with $i \leq m$. Then $n+r-1 \geq i$ and we obtain $r \geq i-n+1$.

(2.1) If $i \geq 2 n-2$ and $i-1 \geq j$, then

$$
\begin{aligned}
j-2 r+2 & \leq(i-1)-2(i-n+1)+2 \\
& =2 n-1-i \\
& \leq 1 .
\end{aligned}
$$

So by (1) above, if $i \geq 2 n-2$ and $i-1 \geq j$, then by (1) we have

$$
b_{j}(P)= \begin{cases}\sum_{k=0}^{l} b_{j-2 k}(X) & \text { if } j=2 l, \\ \sum_{k=0}^{l} b_{j-2 k}(X) & \text { if } j=2 l+1 .\end{cases}
$$

By the same argument as this, if $i \geq 2 n-1$, then we see that

$$
b_{i}(P)= \begin{cases}\sum_{k=0}^{l} b_{i-2 k}(X) & \text { if } i=2 l, \\ \sum_{k=0}^{l} b_{i-2 k}(X) & \text { if } i=2 l+1 .\end{cases}
$$


(2.2) Assume that $i \leq m-1$. If $i=2 n-3$ and $i-1 \geq j$, then $j-2 r+2 \leq 2$. If this equality holds, then $i=n+r-1=m$. But this contradicts the assumption. Hence $j-2 r+2 \leq 1$, and by (1) above we have

$$
b_{j}(P)= \begin{cases}\sum_{k=0}^{l} b_{j-2 k}(X) & \text { if } j=2 l, \\ \sum_{k=0}^{l} b_{j-2 k}(X) & \text { if } j=2 l+1 .\end{cases}
$$

By the same argument as this, if $i \leq m-1, n \geq 1$ (resp. $n \geq 2$ ) and $i=2 n-2($ resp. $i=2 n-3)$, then we see that $b_{2 n-2}(P)=\sum_{k=0}^{n-1} b_{2 n-2-2 k}(X)$ (resp. $b_{2 n-3}(P)=\sum_{k=0}^{n-2} b_{2 n-3-2 k}(X)$ ).

DEFINITION 2.2. Let $X$ be a smooth projective variety of dimension $n$ and let $\mathcal{E}$ be a vector bundle of rank $r$ on $X$.

(1) The Chern polynomial $c_{t}(\mathcal{E})$ is defined by the following:

$$
c_{t}(\mathcal{E})=1+c_{1}(\mathcal{E}) t+c_{2}(\mathcal{E}) t^{2}+\cdots .
$$

(2) For every integer $j$ with $j \geq 0$, the $j$ th Segre class $s_{j}(\mathcal{E})$ of $\mathcal{E}$ is defined by the following equation: $c_{t}\left(\mathcal{E}^{\vee}\right) s_{t}(\mathcal{E})=1$, where $c_{t}\left(\mathcal{E}^{\vee}\right)$ is the Chern polynomial of $\mathcal{E}^{\vee}$ and $s_{t}(\mathcal{E})=\sum_{j \geq 0} s_{j}(\mathcal{E}) t^{j}$.

REMARK 2.3. (1) Let $X$ be a smooth projective variety and let $\mathcal{F}$ be a vector bundle on $X$. Let $\tilde{s}_{j}(\mathcal{F})$ be the Segre class which is defined in [10, Chapter 3]. Then $s_{j}(\mathcal{F})=\tilde{s}_{j}\left(\mathcal{F}^{\vee}\right)$.

(2) For every integer $i$ with $1 \leq i, s_{i}(\mathcal{F})$ can be written by using the Chern classes $c_{j}(\mathcal{F})$ with $1 \leq j \leq i$. (For example, $s_{1}(\mathcal{F})=c_{1}(\mathcal{F})$, $s_{2}(\mathcal{F})=c_{1}(\mathcal{F})^{2}-c_{2}(\mathcal{F})$, and so on.)

DEFINITION 2.3. Let $X$ be a smooth projective variety of dimension $n$ and let $\mathcal{E}$ be an ample vector bundle of rank $n-1$ on $X$. Then the curve genus $\operatorname{cg}(X, \mathcal{E})$ of $(X, \mathcal{E})$ is defined as follows:

$$
c g(X, \mathcal{E})=1+\frac{1}{2}\left(K_{X}+c_{1}(\mathcal{E})\right) c_{n-1}(\mathcal{E}) .
$$

\section{Sectional invariants of scrolls over a smooth projective manifold.}

Theorem 3.1. Let $X, \mathcal{E}, P, H, m$, n and $r$ be as in Notation 2.2. Then the following hold. 
(3.1.1) If $i \geq 2 n-1$, then $e_{i}(P, H)=(i-n+1) c_{n}(X)$.

(3.1.2) If $n \geq 2$, then $e_{2 n-2}(P, H)=(n-1) c_{n}(X)+c_{n}(\mathcal{E})$.

(3.1.3) If $n \geq 3$, then $e_{2 n-3}(P, H)=(n-2)\left(c_{n}(X)-c_{n}(\mathcal{E})\right)-c_{n-1}(\mathcal{E})\left(K_{X}+c_{1}(\mathcal{E})\right)$.

(3.1.4) $e_{1}(P, H)=-(n-2) s_{n}(\mathcal{E})-\left(c_{1}(\mathcal{E})+K_{X}\right) s_{n-1}(\mathcal{E})$.

(3.1.5) $e_{0}(P, H)=s_{n}(\mathcal{E})$.

Proof. By [10, Example 3.2.11] and Remark 2.3 (1), for every integer $l$ with $0 \leq l \leq m$

$$
c_{l}(P)=\sum_{j=0}^{l} \sum_{k=0}^{j}\left(\begin{array}{l}
r-k \\
j-k
\end{array}\right) c_{k}\left(p^{*} \mathcal{E}^{\vee}\right) H(\mathcal{E})^{j-k} c_{l-j}\left(p^{*} \mathcal{T}_{X}\right) .
$$

(Here $p: P \rightarrow X$ denotes the projection.) Hence

$$
\begin{aligned}
& e_{i}(P, H) \\
& =\sum_{j=0}^{i}(-1)^{j}\left(\begin{array}{c}
m-i+j-1 \\
j
\end{array}\right) c_{i-j}(P) H^{m-i+j} \\
& =\sum_{j=0}^{i}(-1)^{j}\left(\begin{array}{c}
m-i+j-1 \\
j
\end{array}\right) \\
& \left\{\sum_{s=0}^{i-j} \sum_{u=0}^{s}\left(\begin{array}{l}
r-u \\
s-u
\end{array}\right) c_{u}\left(p^{*} \mathcal{E}^{\vee}\right) H(\mathcal{E})^{m-i+j-u+s} c_{i-j-s}\left(p^{*} \mathcal{T}_{X}\right)\right\} \\
& =\sum_{\substack{0 \leq k, t \\
0 \leq k+t \leq i}}\left(\sum_{j=0}^{i-t-k}(-1)^{j}\left(\begin{array}{c}
r+j+n-i-2 \\
j
\end{array}\right)\left(\begin{array}{c}
r-k \\
i-t-k-j
\end{array}\right)\right) \\
& c_{k}\left(p^{*} \mathcal{E}^{\vee}\right) c_{t}\left(p^{*} \mathcal{T}_{X}\right) H(\mathcal{E})^{m-k-t} .
\end{aligned}
$$

By Proposition 2.2 and [3, (3.7) in Chapter 0], we get (1) $e_{i}(P, H)$

$$
\begin{aligned}
= & \sum_{\substack{0 \leq k, t \\
0 \leq k+t \leq i}}(-1)^{i-t-k}\left(\begin{array}{c}
k+(n-i-2)+i-t-k \\
i-t-k
\end{array}\right) c_{k}\left(p^{*} \mathcal{E}^{\vee}\right) c_{t}\left(p^{*} \mathcal{T}_{X}\right) H(\mathcal{E})^{m-k-t} \\
= & \sum_{\substack{0 \leq k, t \\
0 \leq k+t \leq i}}(-1)^{i-t-k}\left(\begin{array}{c}
n-t-2 \\
i-t-k
\end{array}\right) c_{k}\left(p^{*} \mathcal{E}^{\vee}\right) c_{t}\left(p^{*} \mathcal{T}_{X}\right) H(\mathcal{E})^{m-k-t} \\
= & \sum_{\substack{0 \leq k, t \\
0 \leq k+t \leq i}}(-1)^{i-t-k}\left(\begin{array}{c}
n-t-2 \\
i-t-k
\end{array}\right) c_{k}\left(\mathcal{E}^{\vee}\right) c_{t}\left(\mathcal{T}_{X}\right) s_{n-k-t}(\mathcal{E})
\end{aligned}
$$


Here we put

$$
E(i, k, t):=(-1)^{i-t-k}\left(\begin{array}{c}
n-t-2 \\
i-t-k
\end{array}\right) c_{k}\left(\mathcal{E}^{\vee}\right) c_{t}\left(\mathcal{T}_{X}\right) s_{n-k-t}(\mathcal{E})
$$

In order to calculate $e_{i}(P, H)$, we have only to consider the case where $E(i, k, t) \neq 0$. We note that if $k+t>n$, then $c_{k}\left(\mathcal{E}^{\vee}\right) c_{t}\left(\mathcal{T}_{X}\right) s_{n-k-t}(\mathcal{E})=0$. So we may assume that $k+t \leq n$.

(a) The case where $i \geq 2 n-1$.

First we note that if $(n, i)=(1,1)$, then

$$
e_{i}(P, H)=e_{1}(P, H)=2-b_{1}(P, H)=2-2 g_{1}(P, H)=2-2 q(X)=c_{1}(X) .
$$

So we assume that $(n, i) \neq(1,1)$.

Here we note that $(n-t-2)-(i-t-k)=n-i-2+k \leq-n+k-1 \leq-1$ and $k+t \leq i$. Hence $\left(\begin{array}{c}n-t-2 \\ i-t-k\end{array}\right) \neq 0$ if and only if one of the following
holds.

(a.1) $i-t-k=0$.

(a.2) $i-t-k>0$ and $n-t-2<0$.

(a.1) If $i-t-k=0$, then $2 n-1 \leq i=t+k \leq n$. Hence $n=1$ and $i=1$. But this contradicts the assumption here.

(a.2) If $i-t-k>0$ and $n-t-2<0$, then $t=n-1$ or $n$ because $k+t \leq n$ and $k \geq 0$. Hence $(t, k)=(n-1,0),(n-1,1)$ or $(n, 0)$.

If $(t, k)=(n-1,0)$, then

$$
\left(\begin{array}{l}
n-t-2 \\
i-t-k
\end{array}\right)=\left(\begin{array}{c}
-1 \\
i-n+1
\end{array}\right)=(-1)^{i-n+1} .
$$

If $(t, k)=(n-1,1)$, then

$$
\left(\begin{array}{c}
n-t-2 \\
i-t-k
\end{array}\right)=\left(\begin{array}{c}
-1 \\
i-n
\end{array}\right)=(-1)^{i-n}
$$

If $(t, k)=(n, 0)$, then

$$
\left(\begin{array}{c}
n-t-2 \\
i-t-k
\end{array}\right)=\left(\begin{array}{c}
-2 \\
i-n
\end{array}\right)=(-1)^{i-n}(i-n+1)
$$


Hence if $(n, i) \neq(1,1)$, then

$$
\begin{aligned}
& e_{i}(P, H) \\
& =(-1)^{2(i-n+1)} c_{n-1}\left(\mathcal{T}_{X}\right) s_{1}(\mathcal{E})+(-1)^{2(i-n)} c_{1}\left(\mathcal{E}^{\vee}\right) c_{n-1}\left(\mathcal{T}_{X}\right) \\
& \quad+(-1)^{2(i-n)}(i-n+1) c_{n}\left(\mathcal{T}_{X}\right) \\
& =(i-n+1) c_{n}(X) .
\end{aligned}
$$

Therefore in any case

$$
e_{i}(P, H)=(i-n+1) c_{n}(X)
$$

if $i \geq 2 n-1$.

(b) The case where $i=2 n-2$. Here we note that $n \geq 2$ in this case.

Then $\left(\begin{array}{c}n-t-2 \\ i-t-k\end{array}\right)=\left(\begin{array}{c}n-t-2 \\ 2 n-t-2-k\end{array}\right)$. Here we note that $(n-t-2) \leq 2 n-t-2-k$. Hence $\left(\begin{array}{c}n-t-2 \\ 2 n-t-2-k\end{array}\right) \neq 0$ if and only if one
of the following holds.

(b.1) $k=n$.

(b.2) $k<n$ and $n-t-2<0$.

(b.1) If $k=n$, then $t=0$ because $t+k \leq n$ and $t \geq 0$, and $\left(\begin{array}{c}n-t-2 \\ 2 n-t-2-k\end{array}\right)=1$. Here we note that $n-t-2 \geq 0$ in this case because $n \geq 2$ and $t=0$. Hence

$$
E(2 n-2, n, 0)=(-1)^{2 n-2-n} c_{n}\left(\mathcal{E}^{\vee}\right)=c_{n}(\mathcal{E}) .
$$

(b.2) If $k<n$ and $n-t-2<0$, then $t=n-1$ or $n$. Hence $(t, k)=(n-1,0)$, $(n-1,1)$ or $(n, 0)$. By the same argument as (a.2), we obtain

$$
\begin{aligned}
E & (2 n-2,0, n-1)+E(2 n-2,1, n-1)+E(2 n-2,0, n) \\
= & (-1)^{2(2 n-2-n+1)} c_{n-1}(X) s_{1}(\mathcal{E})+(-1)^{2(2 n-2-n)} c_{1}\left(\mathcal{E}^{\vee}\right) c_{n-1}(X) \\
& +(-1)^{2(2 n-2-n)}(n-1) c_{n}(X) \\
= & (n-1) c_{n}(X) .
\end{aligned}
$$


Hence we get

$$
e_{2 n-2}(P, H)=(n-1) c_{n}(X)+c_{n}(\mathcal{E}) .
$$

(c) Assume that $i=2 n-3$. Here we note that $n \geq 3$ in this case. Then $\left(\begin{array}{c}n-t-2 \\ 2 n-t-k-3\end{array}\right) \neq 0$ if and only if one of the following holds.

(c.1) $k=n$.

(c.2) $k=n-1$.

(c.3) $k \leq n-2$ and $t>n-2$.

First we consider the case (c. 1 ). Then $t=0$ because $k+t \leq n$ and $t \geq 0$. So we have

$$
\begin{aligned}
E(2 n-3, n, 0) & =(-1)^{2 n-3-0-n}\left(\begin{array}{c}
n-0-2 \\
2 n-3-0-n
\end{array}\right) c_{n}\left(\mathcal{E}^{\vee}\right) \\
& =-\left(\begin{array}{l}
n-2 \\
n-3
\end{array}\right) c_{n}(\mathcal{E}) \\
& =-(n-2) c_{n}(\mathcal{E}) .
\end{aligned}
$$

Next we consider the case (c.2). Then $t=0$ or 1 , and $\left(\begin{array}{c}n-t-2 \\ 2 n-t-k-3\end{array}\right)=1$. Hence we have $E(2 n-3, n-1,0)=-c_{n-1}(\mathcal{E}) s_{1}(\mathcal{E})=$ $=-c_{n-1}(\mathcal{E}) c_{1}(\mathcal{E})$ and $E(2 n-3, n-1,1)=c_{n-1}(\mathcal{E}) c_{1}(X)$.

Finally we consider the case (c.3). Then $(k, t)=(0, n-1),(1, n-1)$ or $(0, n)$. Hence by the same argument as above

$$
E(2 n-3,0, n-1)+E(2 n-3,1, n-1)+E(2 n-3,0, n)=(n-2) c_{n}(X) .
$$

Therefore

$$
\begin{aligned}
& e_{2 n-3}(P, H) \\
& =-(n-2) c_{n}(\mathcal{E})-c_{n-1}(\mathcal{E}) c_{1}(\mathcal{E})+c_{n-1}(\mathcal{E}) c_{1}(X)+(n-2) c_{n}(X) \\
& =(n-2)\left(c_{n}(X)-c_{n}(\mathcal{E})\right)+c_{n-1}(\mathcal{E})\left(c_{1}(X)-c_{1}(\mathcal{E})\right) \\
& =(n-2)\left(c_{n}(X)-c_{n}(\mathcal{E})\right)-c_{n-1}(\mathcal{E})\left(K_{X}+c_{1}(\mathcal{E})\right) .
\end{aligned}
$$


(d) The case where $i=1$. Then by (1) we get

$$
\begin{aligned}
& e_{1}(P, H) \\
& =\sum_{\substack{0 \leq k, t \\
0 \leq k+t \leq 1}}(-1)^{1-t-k}\left(\begin{array}{c}
n-t-2 \\
1-t-k
\end{array}\right) c_{k}\left(\mathcal{E}^{\vee}\right) c_{t}\left(\mathcal{T}_{X}\right) s_{n-k-t}(\mathcal{E}) \\
& =-(n-2) s_{n}(\mathcal{E})+\left(c_{1}\left(\mathcal{E}^{\vee}\right)+c_{1}\left(\mathcal{T}_{X}\right)\right) s_{n-1}(\mathcal{E}) \\
& =-(n-2) s_{n}(\mathcal{E})-\left(c_{1}(\mathcal{E})+K_{X}\right) s_{n-1}(\mathcal{E}) .
\end{aligned}
$$

(e) The case where $i=0$. Then by (1) we get $e_{0}(P, H)=s_{n}(\mathcal{E})$.

We get the assertion of Theorem 3.1.

By Theorem 3.1, we get the following for $n=1$ and 2 .

Corollary 3.1. Let $X, \mathcal{E}, P, H$ and $n$ be as in Notation 2.2. (3.1.1) Assume that $n=1$. Then we get the following:

$$
e_{i}(P, H)= \begin{cases}i(2-2 g(X)) & \text { if } i \geq 1, \\ \operatorname{deg} \mathcal{E} & \text { if } i=0 .\end{cases}
$$

(3.1.2) Assume that $n=2$. Then we get the following:

$$
e_{i}(P, H)= \begin{cases}(i-1) c_{2}(X) & \text { if } i \geq 3, \\ c_{2}(X)+c_{2}(\mathcal{E}) & \text { if } i=2, \\ -\left(c_{1}(\mathcal{E})+K_{X}\right) c_{1}(\mathcal{E}) & \text { if } i=1, \\ s_{2}(\mathcal{E}) & \text { if } i=0 .\end{cases}
$$

TheOREM 3.2. Let $X, \mathcal{E}, P, H, m$, nand $r$ be as in Notation 2.2. Then the following hold.

(3.2.1) If $m \geq i \geq 2 n-1$, then $b_{i}(P, H)=b_{i}(P)$.

(3.2.2) If $n \geq 2$ and $m>2 n-2$, then $b_{2 n-2}(P, H)=b_{2 n-2}(P)+c_{n}(\mathcal{E})-1$.

(3.2.3) If $n \geq 3$ and $m>2 n-3$, then

$$
\begin{aligned}
& b_{2 n-3}(P, H)=b_{2 n-3}(P)+(n-2) c_{n}(\mathcal{E})+c_{n-1}(\mathcal{E})\left(K_{X}+c_{1}(\mathcal{E})\right)+2-2 q(X) \\
& (3.2 .4) b_{1}(P, H)=2+(n-2) s_{n}(\mathcal{E})+\left(c_{1}(\mathcal{E})+K_{X}\right) s_{n-1}(\mathcal{E}) \\
& (3.2 .5) b_{0}(P, H)=s_{n}(\mathcal{E})
\end{aligned}
$$


Proof. Since $e_{i}(P, H)$ has been calculated in Theorem 3.1, we need to study $\sum_{k=0}^{i-1}(-1)^{k} b_{k}(P)$. By using Remark 2.2 , we calculate this.

(a) The case where $i \geq 2 n-1$. Since $b_{m}(P, H)=b_{m}(P)$, we assume that $i<m$.

(a.1) Assume that $i$ is even. Then $i \geq 2 n$. Here we note that $b_{j}(X)=0$ if $j>2 n$. Then

$$
\begin{aligned}
& \sum_{k=0}^{i-1}(-1)^{k} b_{k}(P) \\
& =\sum_{k=0}^{n}\left(\frac{i}{2}-k\right) b_{2 k}(X)-\sum_{k=1}^{n}\left(\frac{i}{2}-k+1\right) b_{2 k-1}(X) \\
& =\sum_{k=0}^{n}(n-k+1) b_{2 k}(X)-\sum_{k=1}^{n}(n-k+2) b_{2 k-1}(X)+\frac{i-2 n-2}{2} e(X) .
\end{aligned}
$$
Here we note that by Remark $2.2(2.1)$, we have $b_{i}(P)=\sum_{k=0}^{n} b_{2 k}(X)$ because
$i \geq 2 n$ in this case. Since $i$ is even, we have

$$
\begin{aligned}
b_{i}(P, H)-b_{i}(P) \\
=(-1)^{i}\left(e_{i}(P, H)-2 \sum_{j=0}^{i-1}(-1)^{j} b_{j}(P)\right)-\sum_{k=0}^{n} b_{2 k}(X) \\
=(i-n+1) c_{n}(X)-2 \sum_{k=0}^{n}(n-k+1) b_{2 k}(X) \\
\quad+2 \sum_{k=1}^{n}(n-k+2) b_{2 k-1}(X)-(i-2 n-2) e(X)-\sum_{k=0}^{n} b_{2 k}(X) \\
=(n+3) e(X)+\sum_{k=0}^{n}(2 k-2 n-3) b_{2 k}(X)+\sum_{k=1}^{n}(2 n-2 k+4) b_{2 k-1}(X) \\
=\sum_{k=0}^{n}(2 k-n) b_{2 k}(X)-\sum_{k=1}^{n}(2 k-n-1) b_{2 k-1}(X) .
\end{aligned}
$$

Here we prove the following.

$$
\text { CLAIM 3.1. (1) } \sum_{k=0}^{n}(2 k-n) b_{2 k}(X)=0 .
$$

(2) $\sum_{k=1}^{n}(2 k-n-1) b_{2 k-1}(X)=0$. 
Proof. (1) Assume that $n=2 l$. Then we note that $(2 k-n) b_{2 k}(X)=0$ if $k=l$. So by Poincaré duality

$$
\begin{aligned}
& \sum_{k=0}^{n}(2 k-n) b_{2 k}(X) \\
& =\sum_{k=0}^{l-1}(2 k-n) b_{2 k}(X)+\sum_{k=l+1}^{n}(2 k-n) b_{2 k}(X) \\
& =\sum_{k=0}^{l-1}(2 k-n) b_{2 k}(X)+\sum_{k=0}^{l-1}(n-2 k) b_{2 n-2 k}(X) \\
& =\sum_{k=0}^{l-1}(2 k-n) b_{2 k}(X)-\sum_{k=0}^{l-1}(2 k-n) b_{2 k}(X) \\
& =0 .
\end{aligned}
$$

If $n=2 l+1$, then by Poincaré duality

$$
\begin{aligned}
& \sum_{k=0}^{n}(2 k-n) b_{2 k}(X) \\
& =\sum_{k=0}^{l}(2 k-n) b_{2 k}(X)+\sum_{k=l+1}^{n}(2 k-n) b_{2 k}(X) \\
& =\sum_{k=0}^{l}(2 k-n) b_{2 k}(X)+\sum_{k=0}^{l}(n-2 k) b_{2 n-2 k}(X) \\
& =\sum_{k=0}^{l}(2 k-n) b_{2 k}(X)-\sum_{k=0}^{l}(2 k-n) b_{2 k}(X) \\
& =0 .
\end{aligned}
$$

Hence we obtain the assertion of (1).

(2) This can be proved by the same argument as (1).

By Claim 3.1, $b_{i}(P, H)=b_{i}(P)$ if $i \geq 2 n-1$ and $i$ is even. 
(a.2) Assume that $i$ is odd. Then

$$
\begin{aligned}
& \sum_{j=0}^{i-1}(-1)^{j} b_{j}(P) \\
& =\sum_{k=0}^{n}\left(\frac{i+1}{2}-k\right) b_{2 k}(X)-\sum_{k=1}^{n}\left(\frac{i-1}{2}-k+1\right) b_{2 k-1}(X) \\
& =\sum_{k=0}^{n}(n-k+1) b_{2 k}(X)-\sum_{k=1}^{n}(n-k+1) b_{2 k-1}(X) \\
& \quad+\left(\frac{i+1}{2}-n-1\right) e(X) .
\end{aligned}
$$

By Remark 2.2 (2.1), we have $b_{i}(P)=\sum_{k=1}^{n} b_{2 k-1}(X)$ because $i \geq 2 n-1$ in this case. Hence by Theorem 3.1 and Claim 3.1, we have

$$
\begin{aligned}
& b_{i}(P, H)-b_{i}(P) \\
& =-\left((i-n+1) e(X)-2 \sum_{j=0}^{i-1}(-1)^{j} b_{j}(P)\right)-b_{i}(P) \\
& =-(n+2) e(X)+\sum_{k=0}^{n}(2 n-2 k+2) b_{2 k}(X)-\sum_{k=1}^{n}(2 n-2 k+3) b_{2 k-1}(X) \\
& =\sum_{k=0}^{n}(n-2 k) b_{2 k}(X)-\sum_{k=1}^{n}(n-2 k+1) b_{2 k-1}(X) \\
& =0 .
\end{aligned}
$$

Therefore $b_{i}(P, H)=b_{i}(P)$ if $i \geq 2 n-1$ and $i$ is odd.

In any case, if $m \geq i \geq 2 n-1$, then $b_{i}(P, H)=b_{i}(P)$.

(b) The case where $i=2 n-2$ and $2 n-2<m$. Then by Remark $2.2(2.1)$ we obtain

$$
b_{j}(P)= \begin{cases}\sum_{k=0}^{l} b_{j-2 k}(X) & \text { if } j=2 l, \\ \sum_{k=0}^{l} b_{j-2 k}(X) & \text { if } j=2 l+1\end{cases}
$$


for every integer $j$ with $j<2 n-2$. Hence

$$
\sum_{j=0}^{2 n-3}(-1)^{j} b_{j}(P)=\sum_{k=0}^{n-2}(n-k-1) b_{2 k}(X)-\sum_{k=1}^{n-1}(n-k) b_{2 k-1}(X) .
$$

By assumption, we have $n+r-1=m \geq i+1=2 n-1$. So by Remark 2.2 (2.2) we obtain

$$
b_{2 n-2}(P)=\sum_{k=0}^{n-1} b_{2 k}(X)
$$

Therefore

$$
\begin{aligned}
b_{2 n-2}(P, H)-b_{2 n-2}(P) & \left(e_{2 n-2}(P, H)-2 \sum_{j=0}^{2 n-3}(-1)^{j} b_{j}(P)\right)-b_{2 n-2}(P) \\
= & (n-1) e(X)+c_{n}(\mathcal{E})-\sum_{k=0}^{n-2}(2 n-2 k-2) b_{2 k}(X) \\
& +\sum_{k=1}^{n-1}(2 n-2 k) b_{2 k-1}(X)-\sum_{k=0}^{n-1} b_{2 k}(X) \\
= & n-1) e(X)+c_{n}(\mathcal{E})-\sum_{k=0}^{n-2}(2 n-2 k-1) b_{2 k}(X) \\
& +\sum_{k=1}^{n-1}(2 n-2 k) b_{2 k-1}(X)-b_{2 n-2}(X) \\
& +\sum_{k=1}^{n}(2 n-2 k+1) b_{2 k-1}(X) . \\
= & (n-1) e(X)+c_{n}(\mathcal{E})+e(X)-1-\sum_{k=0}^{n-1}(2 n-2 k) b_{2 k}(X) \\
& +\sum_{k=1}^{n-1}(2 n-2 k+1) b_{2 k-1}(X)+b_{2 n-1}(X) \\
= & (\mathcal{E})-1-\sum_{k=0}^{n-1}(2 n-2 k) b_{2 k}(X) \\
& \\
& \\
& \\
& \\
& \\
& \\
&
\end{aligned}
$$


Since

$$
\sum_{k=0}^{n-1}(2 n-2 k) b_{2 k}(X)=\sum_{k=0}^{n}(2 n-2 k) b_{2 k}(X)
$$

we obtain

$$
\begin{aligned}
& b_{2 n-2}(P, H)-b_{2 n-2}(P) \\
& =n e(X)+c_{n}(\mathcal{E})-1-\sum_{k=0}^{n}(2 n-2 k) b_{2 k}(X) \\
& \quad+\sum_{k=1}^{n}(2 n-2 k+1) b_{2 k-1}(X) \\
& =c_{n}(\mathcal{E})-1+\sum_{k=0}^{n}(2 k-n) b_{2 k}(X) \\
& \quad-\sum_{k=1}^{n}(2 k-n-1) b_{2 k-1}(X)
\end{aligned}
$$

By Claim 3.1, we get

$$
b_{2 n-2}(P, H)=b_{2 n-2}(P)+c_{n}(\mathcal{E})-1 .
$$

(c) The case where $i=2 n-3$ and $i<m$. Then by Remark 2.2 (2.2) we obtain

$$
b_{j}(P)= \begin{cases}\sum_{k=0}^{l} b_{j-2 k}(X) & \text { if } j=2 l, \\ \sum_{k=0}^{l} b_{j-2 k}(X) & \text { if } j=2 l+1\end{cases}
$$

for every integer $j$ with $j<2 n-3$. Hence

$$
\sum_{j=0}^{2 n-4} b_{j}(P)=\sum_{k=0}^{n-2}(n-k-1) b_{2 k}(X)-\sum_{k=1}^{n-2}(n-k-1) b_{2 k-1}(X) .
$$

By assumption we have $n+r-1=m \geq i+1=2 n-2$. Hence by Remark 2.2 (2.2) we obtain

$$
b_{2 n-3}(P)=\sum_{k=1}^{n-1} b_{2 k-1}(X)
$$


Therefore

$$
\begin{aligned}
& b_{2 n-3}(P, H)-b_{2 n-3}(P) \\
& =-e_{2 n-3}(P, H)+2 \sum_{j=0}^{2 n-4}(-1)^{j} b_{j}(P)-b_{2 n-3}(P) \\
& =-(n-2)\left(e(X)-c_{n}(\mathcal{E})\right)+c_{n-1}(\mathcal{E})\left(K_{X}+c_{1}(\mathcal{E})\right) \\
& +\sum_{k=0}^{n-2}(2 n-2 k-2) b_{2 k}(X)-\sum_{k=1}^{n-2}(2 n-2 k-2) b_{2 k-1}(X) \\
& -\sum_{k=1}^{n-1} b_{2 k-1}(X) \\
& =-(n-2)\left(e(X)-c_{n}(\mathcal{E})\right)+c_{n-1}(\mathcal{E})\left(K_{X}+c_{1}(\mathcal{E})\right) \\
& +\sum_{k=0}^{n-2}(2 n-2 k-2) b_{2 k}(X)-\sum_{k=1}^{n-2}(2 n-2 k-1) b_{2 k-1}(X) \\
& -b_{2 n-3}(X) \\
& =-(n-2)\left(e(X)-c_{n}(\mathcal{E})\right)+c_{n-1}(\mathcal{E})\left(K_{X}+c_{1}(\mathcal{E})\right) \\
& -2 e(X)+2 b_{2 n-2}(X)+2 b_{2 n}(X)-3 b_{2 n-3}(X)-2 b_{2 n-1}(X) \\
& +\sum_{k=0}^{n-2}(2 n-2 k) b_{2 k}(X)-\sum_{k=1}^{n-2}(2 n-2 k+1) b_{2 k-1}(X) \\
& =-(n-2)\left(e(X)-c_{n}(\mathcal{E})\right)+c_{n-1}(\mathcal{E})\left(K_{X}+c_{1}(\mathcal{E})\right) \\
& +\sum_{k=0}^{n}(2 n-2 k) b_{2 k}(X)-\sum_{k=1}^{n}(2 n-2 k+1) b_{2 k-1}(X) \\
& -2 e(X)+2 b_{2 n}(X)-b_{2 n-1}(X) \\
& =-(n-2) e(X)+(n-2) c_{n}(\mathcal{E})+c_{n-1}(\mathcal{E})\left(K_{X}+c_{1}(\mathcal{E})\right) \\
& +\sum_{k=0}^{n}(n-2 k) b_{2 k}(X)-\sum_{k=1}^{n}(n-2 k+1) b_{2 k-1}(X) \\
& +(n-2) e(X)+2 b_{2 n}(X)-b_{2 n-1}(X) .
\end{aligned}
$$


Since $b_{2 n}(X)=1$ and $b_{2 n-1}(X)=b_{1}(X)=2 q(X)$, by Claim 3.1 we obtain

$$
\begin{aligned}
& b_{2 n-3}(P, H)-b_{2 n-3}(P) \\
& =(n-2) c_{n}(\mathcal{E})+c_{n-1}(\mathcal{E})\left(K_{X}+c_{1}(\mathcal{E})\right)+2-2 q(X) .
\end{aligned}
$$

(d) The case where $i=1$.

Then by Theorem 3.1 (3.1.4) and the definition of $b_{1}(P, H)$

$$
\begin{aligned}
b_{1}(P, H) & =-e_{1}(P, H)+2 b_{0}(P) \\
& =2+(n-2) s_{n}(\mathcal{E})+\left(c_{1}(\mathcal{E})+K_{X}\right) s_{n-1}(\mathcal{E}) .
\end{aligned}
$$

(e) The case where $i=0$.

Then by Theorem 3.1 (3.1.5) and the definition of $b_{0}(P, H)$,

$$
b_{0}(P, H)=e_{0}(P, H)=s_{n}(\mathcal{E}) .
$$

Therefore we get the assertion.

Corollary 3.2. Let $X, \mathcal{E}, P, H, m$ and $n$ be as in Notation 2.2. If $n \geq 2$ and $m>i \geq 2 n-2$, then $b_{i}(P, H) \geq b_{i}(P)$.

PRoof. If $i \geq 2 n-1$, then by Theorem 3.2 (3.2.1) we get the assertion. Next we consider the case where $i=2 n-2$. Since $\mathcal{E}$ is ample, we have $c_{n}(\mathcal{E}) \geq 1$. Therefore by Theorem 3.2 (3.2.2), we see $b_{2 n-2}(P, H) \geq b_{2 n-2}(P)$. This completes the proof.

Corollary 3.3. Let $X, \mathcal{E}, P, H, m$ and $n$ be as in Notation 2.2. (3.3.1) Assume that $n=1$. Then we get the following:

$$
b_{i}(P, H)= \begin{cases}b_{i}(P) & \text { if } m \geq i \geq 1, \\ \operatorname{deg} \mathcal{E} & \text { if } i=0 .\end{cases}
$$

(3.3.2) Assume that $n=2$. Then we get the following:

$$
b_{i}(P, H)= \begin{cases}b_{i}(P) & \text { if } m \geq i \geq 3 \\ b_{2}(P)+c_{2}(\mathcal{E})-1 & \text { if } i=2, \\ c_{1}(\mathcal{E})\left(c_{1}(\mathcal{E})+K_{X}\right)+2 & \text { if } i=1 \\ s_{2}(\mathcal{E}) & \text { if } i=0 .\end{cases}
$$

Proof. By Theorem 3.2, we get the assertion. (Here we note that if $n=2$, then $2 n-2=2<n+r-1=m$ because we assume $r \geq 2$ in this paper.) 
REMARK 3.1. Since $\mathcal{E}$ is ample, we see that if $n=1$ or 2 , then $b_{i}(P, H) \geq 0$ for any $i$.

Here we calculate $h_{i}^{j, i-j}(P, H)$ for the case where $m \geq i \geq 2 n-1$.

Theorem 3.3. Let $X, \mathcal{E}, P, H, m$ and $n$ be as in Notation 2.2. If $m \geq i \geq 2 n-1$ and $0 \leq j \leq i$, then $h_{i}^{j, i-j}(P, H)=h^{j, i-j}(P)$.

Proof. First we note that we can take an ample line bundle $A$ on $X$ such that $\mathcal{E} \otimes A^{\otimes t}$ is ample and spanned for every positive integer $t$. Hence $H\left(\mathcal{E} \otimes A^{\otimes t}\right)$ is ample and spanned. We also note that there exists an isomorphism $\phi: \mathbb{P}_{X}\left(\mathcal{E} \otimes A^{\otimes t}\right) \rightarrow P$ with $H\left(\mathcal{E} \otimes A^{\otimes t}\right)=\phi^{*}\left(H \otimes p^{*}\left(A^{\otimes t}\right)\right)$, where $p: P \rightarrow X$ is the projection. Therefore $H \otimes p^{*}\left(A^{\otimes t}\right)$ is also ample and spanned. By this $\phi$, we identify $\mathbb{P}_{X}\left(\mathcal{E} \otimes A^{\otimes t}\right)$ and $P$. By Theorem 3.2 we have

$$
b_{i}\left(P, H\left(\mathcal{E} \otimes A^{\otimes t}\right)\right)=b_{i}(P) .
$$

Hence

$$
\begin{aligned}
& b_{i}\left(P, H \otimes p^{*}\left(A^{\otimes t}\right)\right) \\
& =b_{i}\left(P, H\left(\mathcal{E} \otimes A^{\otimes t}\right)\right) \\
& =b_{i}(P) .
\end{aligned}
$$

On the other hand by Proposition 2.1 (1.1) and (2) we have

$$
h_{i}^{j, i-j}\left(P, H \otimes p^{*}\left(A^{\otimes t}\right)\right)=h^{j, i-j}(P)
$$

because $H \otimes p^{*}\left(A^{\otimes t}\right)$ is ample and spanned. But since $F(t):=$ $:=h_{i}^{j, i-j}\left(P, H \otimes p^{*}\left(A^{\otimes t}\right)\right)-h^{i, i-j}(P)$ is a polynomial in $t$ and $F(t)=0$ for every positive integer $t$, we see that $F(0)=0$, that is,

$$
h_{i}^{j, i-j}(P, H)=h^{i, i-j}(P) .
$$

This completes the proof.

Corollary 3.4. Let $X, \mathcal{E}, P, H, m$ and $n$ be as in Notation 2.2 . (3.4.1) Assume that $n=1$. Then we get the following:

$$
h_{i}^{j, i-j}(P, H)= \begin{cases}h^{j, i-j}(P) & \text { if } m \geq i \geq 1 \text { and } 0 \leq j \leq i, \\ \operatorname{deg} \mathcal{E} & \text { if } i=0 \text { and } j=0 .\end{cases}
$$


(3.4.2) Assume that $n=2$. Then we get the following:

$$
h_{i}^{j, i-j}(P, H)= \begin{cases}h^{j, i-j}(P) & \text { if } m \geq i \geq 3 \text { and } 0 \leq j \leq i, \\ h^{0,2}(P) & \text { if } i=2 \text { and } j=0, \\ h^{2,0}(P) & \text { if } i=2 \text { and } j=2, \\ h^{1,1}(P)+c_{2}(\mathcal{E})-1 & \text { if } i=2 \text { and } j=1, \\ \frac{1}{2}\left(c_{1}(\mathcal{E})\left(c_{1}(\mathcal{E})+K_{X}\right)\right)+1 & \text { if } i=1 \text { and } j=0,1, \\ s_{2}(\mathcal{E}) & \text { if } i=0 \text { and } j=0 .\end{cases}
$$

Proof. If $n=1$, then by Corollary 3.3 and Theorem 3.3 we get the assertion.

Assume that $n=2$. Then by Corollary 3.3 and Theorem 3.3 we get the assertion for the case where $m \geq i \geq 3$ and $0 \leq j \leq i$. If $(i, j)=(2,0)$ (resp. $(2,2)$ ), then by Proposition 2.1 (1.3) and [5, Example $2.10(8)$ ] we have $h_{2}^{0,2}(P, H)=g_{2}(P, H)=h^{2}\left(\mathcal{O}_{P}\right)=h^{0,2}(P) \quad$ (resp. $\quad h_{2}^{2,0}(P, H)=g_{2}(P, H)=$ $\left.=h^{2}\left(\mathcal{O}_{P}\right)=h^{0,2}(P)=h^{2,0}(P)\right)$. Moreover by Corollary 3.3 (3.3.2) and Proposition $2.1(1.1)$ we get $h_{2}^{1,1}(P, H)=h^{1,1}(P)+c_{2}(\mathcal{E})-1$.

Assume that $i=1$. Then by Proposition 2.1 (1.1) we have $b_{1}(P, H)=h_{1}^{1,0}(P, H)+h_{1}^{0,1}(P, H)$. Moreover by Proposition 2.1 (1.2) we have $h_{1}^{1,0}(P, H)=h_{1}^{0,1}(P, H)$. Therefore by Corollary 3.3 we get the assertion for the case $i=1$.

By Corollary 3.3 we get the assertion for the case where $i=0$.

REMARK 3.2. Since $\mathcal{E}$ is ample, we see that if $n=1$ or 2 , then $h_{i}^{j, i-j}(P, H) \geq 0$ for any $i$ and $j$ with $0 \leq j \leq i \leq m$.

\section{A new invariant of generalized polarized manifolds.}

Here we use Notation 2.2. Assume that $i=2 n-3, n \geq 3$ and $i<m$. Then by Theorem $3.2(3.2 .3)$ we see that $(n-2) c_{n}(\mathcal{E})+c_{n-1}(\mathcal{E})\left(K_{X}+c_{1}(\mathcal{E})\right)$ is even because $b_{2 n-3}(P, H)$ and $b_{2 n-3}(P)$ are even (see [8, Theorem 3.1 (3.1.2)]). We put

$$
v(X, \mathcal{E}):=1+\frac{1}{2}\left((n-2) c_{n}(\mathcal{E})+c_{n-1}(\mathcal{E})\left(K_{X}+c_{1}(\mathcal{E})\right)\right)
$$

Here we note that $r \geq n-1$ since $n+r-1=m \geq i+1=2 n-2$.

If $r=n-1$, then

$$
v(X, \mathcal{E})=1+\frac{1}{2}\left(K_{X}+c_{1}(\mathcal{E})\right) c_{n-1}(\mathcal{E}) .
$$


So $v(X, \mathcal{E})$ is thought to be a generalization of the curve genus $\operatorname{cg}(X, \mathcal{E})$ of $(X, \mathcal{E})$ (see Definition 2.3). Here we define $v(X, \mathcal{E})$ again.

DEFinition 4.1. Let $(X, \mathcal{E})$ be a generalized polarized manifold of dimension $n \geq 3$. Assume that $r \geq n-1$. Then the invariant $v(X, \mathcal{E})$ of $(X, \mathcal{E})$ is defined as follows.

$$
v(X, \mathcal{E}):=1+\frac{1}{2}\left((n-2) c_{n}(\mathcal{E})+c_{n-1}(\mathcal{E})\left(K_{X}+c_{1}(\mathcal{E})\right)\right) .
$$

Since $b_{2 n-3}(P, H)-b_{2 n-3}(P)=2 v(X, \mathcal{E})-2 q(X)$ by Theorem 3.2 (3.2.3), we can propose the following conjecture.

CONJECTURE 4.1. Let $(X, \mathcal{E})$ be a generalized polarized manifold of dimension $n \geq 3$. Assume that $r \geq n-1$. Then $v(X, \mathcal{E}) \geq q(X)$.

Here we study the non-negativity of $v(X, \mathcal{E})$.

THEOREM 4.1. Let $(X, \mathcal{E})$ be a generalized polarized manifold of dimension $n \geq 3$. Assume that $r \geq n-1$. Then $v(X, \mathcal{E}) \geq 0$.

Proof. If $r=n-1$, then $v(X, \mathcal{E})$ is the curve genus and then $v(X, \mathcal{E}) \geq 0$ by [19, Theorem 1]. So we may assume that $r \geq n$.

If $r \geq n$ and $K_{X}+c_{1}(\mathcal{E})$ is nef, then $\left(K_{X}+c_{1}(\mathcal{E})\right) c_{n-1}(\mathcal{E}) \geq 0$ because $\mathcal{E}$ is ample. Furthermore $c_{n}(\mathcal{E}) \geq 1$. So we obtain $v(X, \mathcal{E}) \geq 2$.

If $r \geq n$ and $K_{X}+c_{1}(\mathcal{E})$ is not nef, then $(X, \mathcal{E}) \cong\left(\mathbb{P}^{n}, \mathcal{O}_{\mathbb{P}^{n}}(1)^{\oplus n}\right)$ by [22, Theorem 1 and Theorem 2]. In this case $\left(K_{X}+c_{1}(\mathcal{E})\right) c_{n-1}(\mathcal{E})=-n$ and $c_{n}(\mathcal{E})=1$. Hence $v(X, \mathcal{E})=0$. Therefore we get the assertion.

THEOREM 4.2. Let $(X, \mathcal{E})$ be a generalized polarized manifold of $d i$ mension $n \geq 3$. Assume that $r \geq n-1$.

(1) If $v(X, \mathcal{E})=0$, then $(X, \mathcal{E})$ is one of the following.

(1.1) $\left(\mathbb{P}^{n}, \mathcal{O}_{\mathbb{P}^{n}}(1)^{\oplus n}\right)$.

(1.2) $\left(\mathbb{P}^{n}, \mathcal{O}_{\mathbb{P}^{n}}(1)^{\oplus n-1}\right)$.

(1.3) $\left(\mathbb{P}^{n}, \mathcal{O}_{\mathbb{P}^{n}}(1)^{\oplus n-2} \oplus \mathcal{O}_{\mathbb{P}^{n}}(2)\right)$.

(1.4) $\left(\mathrm{Q}^{n}, \mathcal{O}_{\mathrm{Q}^{n}}(1)^{\oplus n-1}\right)$.

(1.5) $X \cong \mathbb{P}_{\mathrm{P}^{1}}(\mathcal{F})$ for some vector bundle $\mathcal{F}$ of rank $n$ on $\mathrm{P}^{1}$, and $\mathcal{E} \cong \oplus_{j=1}^{n-1}\left(H(\mathcal{F})+\pi^{*} \mathcal{O}_{\mathrm{P}^{1}}\left(b_{j}\right)\right)$, where $\pi: X \rightarrow \mathbb{P}^{1}$ is the bundle projection. 
(2) If $v(X, \mathcal{E})=1$, then $(X, \mathcal{E})$ is one of the following.

(2.1) $X \cong \mathbb{P}_{C}(\mathcal{F})$ for some vector bundle $\mathcal{F}$ of rank $n$ on a smooth elliptic curve $C$, and $\mathcal{E}_{F} \cong \mathcal{O}_{\mathrm{P}^{n-1}}(1)^{\oplus n-1}$ for any fiber $F$ of the bundle projection $X \rightarrow C$.

(2.2) $K_{X}+\operatorname{det}(\mathcal{E})=\mathcal{O}_{X}$ and $r=n-1$.

Proof. If $v(X, \mathcal{E}) \leq 1$, then by the proof of Theorem 4.1, one of the following cases occurs:

(4.2.1) $r=n-1$.

(4.2.2) $r \geq n$ and $K_{X}+c_{1}(\mathcal{E})$ is not nef.

If $r=n-1$ and $v(X, \mathcal{E})=0$ (resp. $v(X, \mathcal{E})=1$ ), then $0=v(X, \mathcal{E})=$ $=c g(X, \mathcal{E})(\operatorname{resp} .1=v(X, \mathcal{E})=c g(X, \mathcal{E}))$ and by $[19$, Theorem 1 and Theorem 2 ] we get the above types.

If $r \geq n$ and $K_{X}+c_{1}(\mathcal{E})$ is not nef, then $(X, \mathcal{E}) \cong\left(\mathbb{P}^{n}, \mathcal{O}_{\mathbb{P}^{n}}(1)^{\oplus n}\right)$ by [22, Theorem 1 and Theorem 2] and then $v(X, \mathcal{E})=0$. Hence we get the assertion.

REMARK 4.1. (1) If $(X, \mathcal{E})$ is the type (2.2) in Theorem 4.2, then a classification of $(X, \mathcal{E})$ has been obtained by [20].

(2) If $v(X, \mathcal{E}) \leq 1$, then we see that $v(X, \mathcal{E}) \geq q(X)$.

Proposition 4.1. Let $(X, \mathcal{E})$ be a generalized polarized manifold of dimension $n \geq 3$. Assume that $r \geq n-1$ and $\mathcal{E}$ is spanned. Then $v(X, \mathcal{E}) \geq q(X)$.

Proof. Set $(P, H):=\left(\mathbb{P}_{X}(\mathcal{E}), H(\mathcal{E})\right)$. Then $H$ is ample and spanned. So by Proposition 2.1 (2.1) we obtain

$$
b_{2 n-3}(P, H) \geq b_{2 n-3}(P) .
$$

On the other hand

$$
\begin{aligned}
& b_{2 n-3}(P, H)-b_{2 n-3}(P) \\
& =2 v(X, \mathcal{E})-2 q(X) .
\end{aligned}
$$

Hence we get the assertion.

REMARK 4.2. (1) By Theorem 4.2 we can determine the type of $(X, \mathcal{E})$ such that $v(X, \mathcal{E})=q(X)$ and $q(X) \leq 1$. 
(2) For every integer $q \geq 2$, there exists an example of $(X, \mathcal{E})$ with $v(X, \mathcal{E})=q=q(X)$ (This was given by the referee.): Let $C$ be a smooth projective curve with $g(C)=q \geq 2$. We note that there exist vector bundles $\mathcal{F}$ and $\mathcal{G}$ on $C$ with rank $\mathcal{F}=n$ and rank $\mathcal{G}=n-1$ such that $\mathcal{E}:=H(\mathcal{F}) \otimes \pi^{*}(\mathcal{G})$ is an ample vector bundle of rank $n-1$ on $X$, where $X:=\mathbb{P}_{C}(\mathcal{F}), H(\mathcal{F})$ is the tautological line bundle of $\mathcal{F}$ and $\pi: X \rightarrow C$ is the bundle projection. Then we can easily check that $v(X, \mathcal{E})=c g(X, \mathcal{E})=q \geq 2$.

(3) We see that if $\mathcal{E}$ is ample and spanned, rank $\mathcal{E}=n-1$ and $v(X, \mathcal{E})=q(X) \geq 2$, then $(X, \mathcal{E})$ is isomorphic to the type in (2) above. This has been proved by [17, Theorem].

Here we give the following conjecture which was pointed out by the referee:

CONJECTURE 4.2. Let $X$ be a smooth projective variety of dimension $n \geq 3$ and let $\mathcal{E}$ be an ample vector bundle on $X$ with rank $\mathcal{E}=r$. Assume that $r \geq n-1$ and $q(X) \geq 2$. If $\mathcal{E}$ is spanned and $v(X, \mathcal{E})=q(X)$, then $r=n-1$.

REMARK 4.3. We consider the case where $r \geq n \geq 3$. (The following (a) and (b) were also pointed out by the referee.)

(a) If $K_{X}+c_{1}(\mathcal{E})$ is not ample, then by [4, Main Theorem] $(X, \mathcal{E})$ is one of the following 6 types and we can calculate $v(X, \mathcal{E})$ and $q(X)$ :

(a.1) $\left(\mathbb{P}^{n}, \mathcal{O}_{\mathrm{P}^{n}}(1)^{\oplus n+1}\right)$. In this case $(v(X, \mathcal{E}), q(X))=(1+(1 / 2)$. $(n-2)(n+1), 0)$.

(a.2) $\left(\mathbb{P}^{n}, \mathcal{O}_{\mathrm{P}^{n}}(1)^{\oplus n}\right)$. In this case $(v(X, \mathcal{E}), q(X))=(0,0)$.

(a.3) $X \cong \mathbb{P}_{C}(\mathcal{F})$ for a vector bundle $\mathcal{F}$ of rank $n$ on a smooth curve $C$, and $\mathcal{E} \cong H(\mathcal{F}) \otimes \pi^{*}(\mathcal{G})$ for a vector bundle $\mathcal{G}$ on $C$ with rank $\mathcal{G}=n$, where $\pi: X \rightarrow C$ is the bundle projection. In this case $(v(X, \mathcal{E}), q(X))=\left(g(C)+(n-1)\left(g(C)-1+c_{1}(\mathcal{F})+c_{1}(\mathcal{G})\right)\right.$, $g(C))$.

(a.4) $\left(\mathbb{P}^{n}, \mathcal{O}_{\mathrm{p}^{n}}(1)^{\oplus n-1} \oplus \mathcal{O}_{\mathbb{P}^{n}}(2)\right)$. In this $\quad$ case $(v(X, \mathcal{E}), q(X))=$ $=(n-1,0)$.

(a.5) $\left(\mathbb{P}^{n}, \mathcal{T}_{\mathrm{p}^{n}}\right)$. In this case $(v(X, \mathcal{E}), q(X))=(1+(1 / 2)(n-2)$. $(n+1), 0)$.

(a.6) $\left(\mathrm{Q}^{n}, \mathcal{O}_{\mathrm{Q}^{n}}(1)^{\oplus n}\right)$. In this case $(v(X, \mathcal{E}), q(X))=(n-1,0)$.

Here we consider the case where $(X, \mathcal{E})$ is the type (a.3) above. Then $1 \leq c_{n}(\mathcal{E})=c_{1}(\mathcal{F})+c_{1}(\mathcal{G})$ because $\mathcal{E}$ is ample. Hence $g(C)-1+c_{1}(\mathcal{F})+$ 
$+c_{1}(\mathcal{G}) \geq 0$ and we get $v(X, \mathcal{E}) \geq g(C)=q(X)$. Moreover if $g(C) \geq 1$, then we see that $v(X, \mathcal{E}) \geq q(X)+(n-1)>q(X)$.

We also note that if $\mathcal{E}$ is spanned by global sections, then $c_{n}(\mathcal{E}) \geq 2$ by [21, (3.4) Theorem] and we obtain $v(X, \mathcal{E}) \geq q(X)+(n-1)>q(X)$.

(b) Next we assume that $K_{X}+c_{1}(\mathcal{E})$ is ample. Then we can prove the following:

Proposition 4.2. (1) If $K_{X}+c_{1}(\mathcal{E})$ is ample, then $v(X, \mathcal{E}) \geq 1+$ $+\frac{1}{2}(n-1)$

(2) If $K_{X}+c_{1}(\mathcal{E})$ is ample and $\mathcal{E}$ is spanned, then $v(X, \mathcal{E}) \geq n$.

Proof. (1) By assumption, we have $c_{n}(\mathcal{E}) \geq 1$ and $\left(K_{X}+c_{1}(\mathcal{E})\right) c_{n-1}(\mathcal{E}) \geq 1$. Hence we get the assertion of (1).

(2) Since $\mathcal{E}$ is spanned and $K_{X}+c_{1}(\mathcal{E})$ is ample, we see that $c_{n}(\mathcal{E}) \geq 2$ by $\left[21, \quad(3.4)\right.$ Theorem]. Hence $(n-2) c_{n}(\mathcal{E})+\left(K_{X}+c_{1}(\mathcal{E})\right) c_{n-1}(\mathcal{E}) \geq$ $\geq 2(n-2)+2$ because the term on the left is even. Therefore we get the assertion of (2).

The above results suggest that for the case where $r \geq n$ and $v(X, \mathcal{E}) \neq q(X)$ there are some gaps for the value of $v(X, \mathcal{E})-q(X)$, depending on $n$. We will investigate this in a future paper.

\section{REFERENCES}

[1] E. BALLICO, On vector bundles on 3-folds with sectional genus 1, Trans. Amer. Math. Soc., 324 (1991), pp. 135-147.

[2] T. FuJita, Ample vector bundles of small $c_{1}$-sectional genera, J. Math. Kyoto Univ., 29 (1989), pp. 1-16.

[3] T. Fujita, Classification Theories of Polarized Varieties, London Math. Soc. Lecture Note Ser. 155, Cambridge University Press, (1990).

[4] T. FuJita, On adjoint bundles of ample vector bundles, Complex algebraic varieties (Bayreuth, 1990), pp. 105-112, Lecture Notes in Math., 1507, Springer, Berlin, 1992.

[5] Y. Fukuma, On the sectional geometric genus of quasi-polarized varieties, I, Comm. Alg., 32 (2004), pp. 1069-1100.

[6] Y. Fukuma, Problems on the second sectional invariants of polarized manifolds, Mem. Fac. Sci. Kochi Univ. Ser. A Math., 25 (2004), pp. 55-64.

[7] Y. FuKuma, On the second sectional H-arithmetic genus of polarized manifolds, Math. Z., 250 (2005), pp. 573-597.

[8] Y. Fukuma, On the sectional invariants of polarized manifolds, J. Pure Appl. Alg., 209 (2007), pp. 99-117. 
[9] Y. Funuma - H. Ishihara, A generalization of curve genus for ample vector bundles, II, Pacific J. Math., 193 (2000), pp. 307-326.

[10] W. Fulton, Intersection Theory, Ergebnisse der Mathematik und ihrer Grenzgebiete, 2 (1984), Springer-Verlag.

[11] D. Fusi - A. LANTERI, Ample vector bundles with small $g-q$, Comm. Alg., 34 (2006), pp. 2989-3008.

[12] F. HiRzEBRuch, Topological methods in algebraic geometry, Grundlehren der mathematischen Wissenschaften, 131 Springer-Verlag, 1966.

[13] P. Ionescu, Embedded projective varieties of small invariants, in Proceedings of the Week of Algebraic Geometry, Bucharest 1982, Lecture Notes in Math., 1056 (1984), pp. 142-186.

[14] P. Ionescu, Embedded projective varieties of small invariants, II, Rev. Roumanie Math. Puves Appl., 31 (1986), pp. 539-544.

[15] P. Ionescu, Embedded projective varieties of small invariants, III, in Algebraic Geometry, Proceedings of Conference on Hyperplane Sections, L’Aquila, Italy, 1988 Lecture Notes in Math., 1417 (1990), pp. 138-154.

[16] H. IshiHARA, A generalization of curve genus for ample vector bundles, I, Comm. Alg., 27 (1999), pp. 4327-4335.

[17] A. LANTERI - H. MAEDA - A. J. Sommese, Ample and spanned vector bundles of minimal curve genus, Arch. Math., 66 (1996), pp. 141-149.

[18] A. LANTERI - D. Struppa, Projective manifolds whose topology is strongly reflected in their hyperplane sections, Geom. Dedicata, 21 (1986) pp. 357-374.

[19] H. MAEDA, Ample vector bundles of small curve genera, Arch. Math., 70 (1998) pp. 239-243.

[20] T. Peternell - M. Szurek - J. A. Wiśniewski, Fano manifolds and vector bundles, Math. Ann., 294 (1992) pp. 151-165.

[21] J. A. WIŚNIEWsKI, Length of extremal rays and generalized adjunction, Math. Z., 200 (1989), pp. 409-427.

[22] Y. G. YE - Q. ZHANG, On ample vector bundles whose adjoint bundles are not numerically effective, Duke Math. J., 60 (1990) pp. 671-687.

Manoscritto pervenuto in redazione il 10 luglio 2007. 
